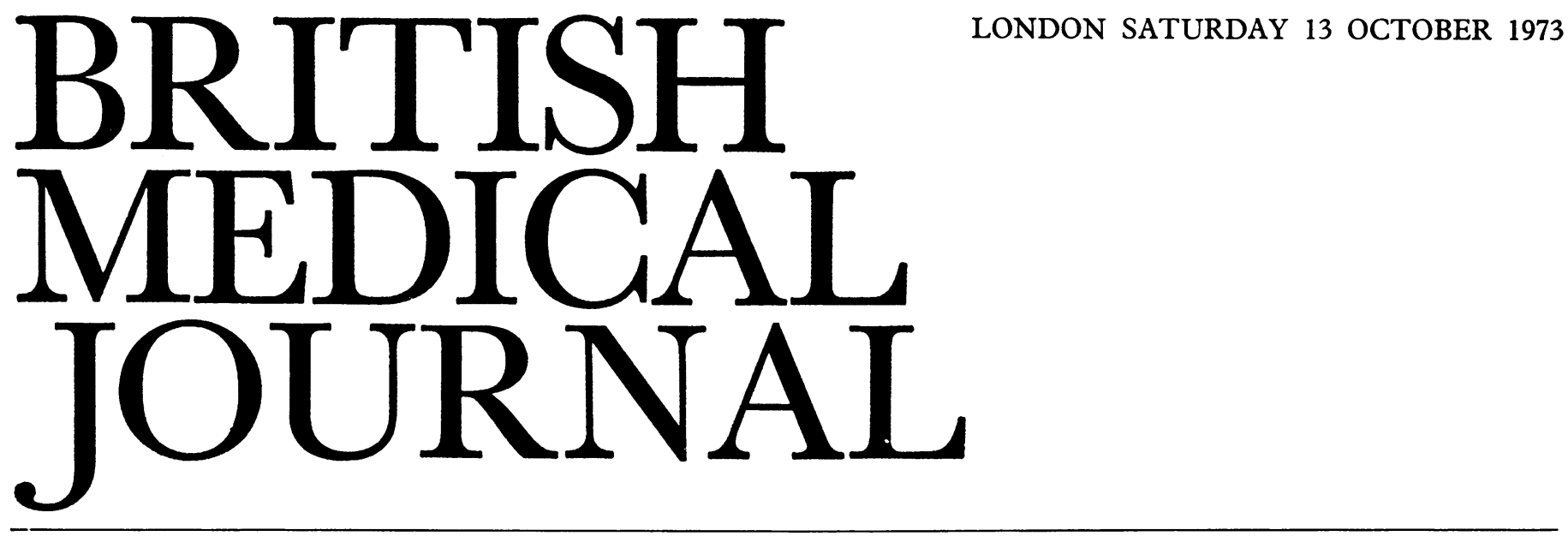

\title{
Deliberate Injury of Children
}

Child abuse is a major problem for accident and emergency departments, children's hospitals, general practitioners, social workers, the police, and many others concerned with the welfare of children. The injuries seen include fractures of the skull, clavicles, ribs, or long bones; bruises, lacerations, and soft tissue swelling; burns-for example, on the buttocks, by sitting the child on an electric radiator-or elsewhere by cigarettes; subdural effusion, as a result of direct injury to the skull or by vigorous shaking ${ }^{1}$; damage to the liver or perforation of the stomach or intestine; emotional deprivation, starvation, dehydration, anaemia, growth retardation, or poisoning. A child may be poisoned by deliberate administration of a drug, by giving an overdose, or by deliberately leaving poisonous material conveniently near him.

Most victims of child abuse are under 4 years old and many under 2. How prevalent these serious assaults are is unknown. A figure of 4,600 cases a year in the U.K. has been given. ${ }^{2}$ Six children per 1,000 live births is another estimate. ${ }^{3} \mathrm{C}$. H. Kempe estimated that it was the cause of $10 \%$ of all casualties under 2 and $25 \%$ of all fractures under that age. ${ }^{3}$ A general practitioner might on average see a case every five years.

Some 30 to $60 \%$ of parent batterers were themselves battered as children. ${ }^{4}$ The battering may be due to loss of temper or to an attempt to discipline the child, especially when inhibitions have been lost under the effect of alcohol. Perhaps $5 \%$ of batterers are psychotic ${ }^{3}$ and more of them aggressive psychopaths; but the majority are more or less normal parents who are worn out by their small children, parents who are lonely, poorly, immature, or under emotional or financial stress, with no one to turn to for help. The batterer is usually the mother or father, but sometimes a grandparent or other children. ${ }^{5}$ If one parent batters the child, the other one almost certainly knows about it and aids and abets. ${ }^{3}$

The diagnosis can be difficult to reach and impossible to prove. There may be a suspicious delay between an injury and the visit to the doctor. The story given may not agree with the physical findings and the explanation of injuries may be vague. The parents may show inappropriate agitation or evidence of rejection, bitterly describing the child's constant crying or his backwardness, which examination fails to confirm. Repeated visits to the family doctor or hospital may arouse suspicion. The parents often deliberately conceal their child's injuries, merely consulting the doctor about feeding or sleep problems, crying, skin lesions, poor weight gain, or abdominal pain. They may claim that the child bruises easily. A history of injuries or possible injuries to other children is important.
Suspicious features are injuries in different stages of resolution; bruises for which there is no good explanation, especially on the face; bite marks, a torn frenulum linguae, owing to the bottle or a fist being forced into the mouth; petechial haemorrhage in the skin folds at the root of the neck or elsewhere; or the child may be distinctly apathetic. ${ }^{6}$ If subdural effusion is present, there are almost always retinal haemorrhages. The specialist has to eliminate other diseases, such as coagulation defects in the case of bruising, and a wide variety of conditions if the baby seems merely to be failing to thrive. The first investigation in hospital when child abuse is suspected is an $x$-ray examination of the skeleton for fractures, periosteal elevation, and other changes, and the $x$-raying should be repeated in two or three weeks, partly to determine whether new lesions have appeared.

The management of this condition can be a difficult and time-consuming matter. The family doctor's first and immediate responsibility is to refer the child to the nearest hospital for investigation without delay, for delay may mean the death of the child. A working party, the Tunbridge Wells Study Group, under the auspices of the Spastics' Society and with the help of the Department of Health and Social Security has just published an excellent report ${ }^{2}$ on the problem. Its conclusions are summarized in our Medical Practice section this week. The report is the product of meetings of doctors, a magistrate, lawyers, a health visitor, social workers, and representatives of the police, the National Society for the Prevention of Cruelty to Children, and other organizations. It emphasizes that rehabilitation of the family is more important to society than punishment and that battering parents need help and not condemnation. It also brings out the importance of team work between the hospital, family doctor, social services, and police and condemns precipitate unilateral action. For instance, while admission to hospital is essential, the child should not be discharged without the agreement of all concerned. The report recommends that the health visitor should always be told of cases of child abuse, and about poisonings whether accidental or not, and the social service should always be told too, even though there is nothing more than suspicion. Though doctors dislike the idea of involving the police, fearing they might be breaking their patient's confidence or lest police interrogation might result and so make further help impossible, co-operation with the police is strongly advised. But the advisability of it will vary from place to place, and will depend in part on the attitude of the chief constable. Other recommendations include case conferences between all concerned and the setting up of an 
area review committee, to include a family doctor, probation officer, and representatives of other relevant bodies.

The report emphasizes the key role of the consultant in accident and emergency departments in making the diagnosis, collecting data, and ensuring that attendances at different hospitals and different parts of one hospital are recorded, so that the suspicion of child abuse can be aroused. For a battering family may go from hospital to hospital, or from one department of a hospital to another, turning up at one time with the child burnt, another time with a fracture, and another time with failure to thrive. As the report points out, research is needed into the effect of different types of management, including criminal proceedings. Methods of prevention also need study. They might include better family planning, the protection of siblings (who are at considerable risk), and instruction in schools about the development and needs of children.

The recommendations are eminently sensible. Increases in staff will be needed to carry out some of them. But children's lives are at stake.

1 Caffey, J., American Fournal of Diseases of Children, 1972, 124, 161.

2 Tunbridge Wells Study Group, Non-accidental Injury to Children, 1973.

3 Kempe, C. H., Archives of Disease in Childhood, 1971, 46, 28.

4 Solomon, T., Symposium on Child Abuse, Pediatrics, Supplement 51, No. 4 , pt. 2,773 .

5 Adelson, 2, 77.

${ }^{6}$ Medical Aspects of Home Hazards, ed. A. Nelson, London, Medical

Commission on Accident Prevention, 1972.

\section{Siøgren's Syndrome}

"Commoner than hitherto realized" is a hard-worked cliché in medical writing, often enthusiastically applied to descriptions of clinical rarities. But a condition for which it appears to be well justified is Sjøgren's syndrome, first clearly described by Henriks Sjøgren, a Swedish ophthalmologist, in $1933 .{ }^{1}$ It is defined as a triad of dry mouth (xerostomia), dry eyes (xerophthalmia), and a connective tissue disease-usually rheumatoid arthritis.

During the past decade the extent to which the disease affects a variety of organs has become apparent. ${ }^{2-4}$ The characteristic lymphoid infiltration is not confined to the salivary and lachrymal glands but occurs also in the nasal cavity, pharynx, larynx, trachea, bronchi, sweat glands, and kidney, with such diverse effects as recurrent epistaxis, bronchitis, recurrent chest infections, and renal tubular abnormalities as well as the better known ocular and oral symptoms.

A characteristic of the syndrome is the presence of hypergammaglobulinaemia and of a variety of circulating autoantibodies, both organ-specific and non-organ-specific. Its clinical and immunological features have recently been reviewed in the B.M.F. ${ }^{5}$ and in a monograph by M. A. Shearn. ${ }^{6}$ The exact prevalence of the syndrome and in particular the extent of its relationship with rheumatoid arthritis are not precisely known. Most series to date have been highly selected and have concentrated on the more florid forms of the disease.

More work on it has now been reported in two articles by $\mathrm{K}$. Whaley and his colleagues ${ }^{7}$ from Glasgow in a six-year study of 171 patients with Sjøgren's syndrome. Of these, 94 patients had rheumatoid arthritis, 4 had systemic lupus erythematosus, 1 had progressive systemic sclerosis, and 1 had psoriatic arthritis. In 71 patients Sjøgren's syndrome was unassociated with rheumatoid arthritis or other connective tissue or rheumatic diseases (the so-called sicca syndrome).
The mean age was 57 years and $89 \%$ were female-a sex incidence closer to that of systemic lupus erythematosus than rheumatoid arthritis.

Some interesting clinical points emerged. While the majority of patients complained of ocular symptoms, few were diagnosed by their general practitioner or consultant physician as having the disease. The symptoms of keratoconjunctivitis sicca were commoner in patients with the sicca syndrome than in those with rheumatoid arthritis, the commonest complaints being sensations of "burning" or a "foreign body." While the diagnostic value of the Schirmer test for secretion of tears was stressed, diminished tear secretion was also found in hot dry atmospheres and with increasing frequency in the elderly. In elderly patients the keratoconjunctivitis sicca does not appear to reflect subclinical inflammation of the lachrymal glands and is not associated with the presence of circulating autoantibodies. ${ }^{8}$ Artificial tear drops ( $0.5 \%$ carboxymethylcellulose) were satisfactory in controlling ocular symptoms in most of the patients.

The symptoms and signs of xerostomia were more variable and were often intermittent. A history of increased intake of fluid was common. The diagnostic value of biopsy of labial salivary glands was again confirmed. ${ }^{9}$ Lymphocytic inflammation of the salivary gland was found in $62 \%$ of the patients with the sicca syndrome, $70 \%$ of patients with rheumatoid arthritis and Sjøgren's syndrome, and, notably, in 19\% of patients with rheumatoid arthritis alone. Enlargement of the salivary glands was seen in 37 patients. The parotid glands were most frequently affected, and unilateral and intermittent swelling was common. Rates of salivary flow were reduced in all groups of patients with this syndrome, but the flow rate is limited as an index of salivary gland function because the variation is so wide in normal persons.

The study re-emphasized how commonly the upper respiratory tract is affected in this disease. Whaley and his colleagues suggest that postoperative suppurative parotitis might be more prevalent in patients with Sjøgren's syndrome than in general. On similar reasoning it would also seem likely that postoperative atelectasis and chest infection are commoner in this group of patients.

Of 15 patients assessed for renal tubular function one had overt renal tubular acidosis, five had latent renal tubular acidosis, and three had impaired renal concentrating ability. One patient had generalized aminoaciduria.

Immunological studies again confirmed the high incidence of non-organ-specific and of organ-specific antibodies, though it is interesting to note that there was no clear association between organ-specific autoimmune diseases and Sjogren's syndrome.

G. R. V. Hughes and Whaley have advocated the routine testing of patients with rheumatic diseases for evidence of conjunctivitis sicca. ${ }^{5}$ Apart from the considerable disability associated with Sjøgren's syndrome two other features deserve particular attention. Over the past two decades some cases of malignant disease of the reticuloendothelial system have been reported in association with it. ${ }^{10-12}$ The incidence is unknown, though rare, and this complication appears to be more common in patients with the sicca syndrome alone. Of interest was the finding that of the 7 patients in the Glasgow series with disease of the lachrymal glands one developed lymphosarcoma. The only patient in an earlier series ${ }^{3}$ who had enlarged lachrymal glands subsequently developed reticulum cell sarcoma. The second feature to be noted is the tendency of these patients to develop drug allergies. B. O. Williams and colleagues $^{13}$ noted that the incidence of penicillin allergy in patients with Sjøgren's syndrome was $40 \%$ as compared with 